\title{
Two Techniques for the Efficient Numerical Calculation of the Green's Functions for Planar Shielded Circuits and Antennas
}

\author{
Alejandro Alvarez-Melcón and Juan R. Mosig, Fellow, IEEE
}

\begin{abstract}
In this paper we present new contributions to the computation of the Green's functions arising in the analysis of multilayered shielded printed circuits and antennas. First the quasistatic term of the spectral domain Green's functions is extracted so that the convergence of the reminder dynamic modal series is enhanced. Moreover, it is shown that by extracting a second-order quasi-static term the convergence is further improved. In regard to the quasi-static terms they are computed in the spatial domain by numerically evaluating the associated spatial images series. Then a new and efficient technique is developed for the summation of the slowly convergent modal series. The technique can be viewed as the application of the integration by parts technique to discrete sequences and greatly accelerates the convergence rate of the series involved. It is shown that the new algorithm is numerically very robust and leads to a drastic reduction in the computational effort and time usually required for the numerical evaluation of the shielded Green's functions.
\end{abstract}

Index Terms-Boxed circuits, convergence acceleration, Green's functions, infinite series, integral equation, multilayer media, shielded circuits.

\section{INTRODUCTION}

$\mathbf{T}$ HE analysis of shielded microwave circuits and cavity backed antennas is a subject that has always attracted attention, and numerous numerical models have been developed in the past [1]-[15]. Moreover, the interest in the full wave electromagnetic analysis of such components has recently grown, especially since the advent of monolithic microwave integrated circuits (MMIC). The high degree of integration level achieved implies that all the subelements of the circuit are disposed to be very tightly closed together, and electromagnetic coupling cannot be neglected. The analysis of these elements separately with subsequent interconnection, or the use of quasi-static approaches are, therefore, not appropriate for such complex circuits. In addition, the use of suitable packaging in MMIC circuits to provide isolation is now general, and this results in that the corresponding circuits need to be modeled taking into account the surrounding enclosure. Also a metallic enclosure gives the mechanical support to many antenna systems and it improves heat dissipation [16]. Related to the analysis of boxed circuits, of particular interest are the works [12] and [15] which studied the effects of the shielding enclosure in the

Manuscript received May 8, 1998.

The authors are with the Electromagnetics and Acoustics Laboratory (LEMA), Swiss Federal Institute of Technology (EPFL), Lausanne, Switzerland.

Publisher Item Identifier S 0018-9480(00)07391-9. performances of various circuits and established the conditions under which shielding must be taken into account because it strongly modifies the electrical behavior of the structures.

For the analysis of shielded circuits and antennas the finite elements method has been successfully used in the past [1]-[4], but it usually leads to computer codes which are very heavy and time-consuming. Perhaps the most popular technique in the analysis of printed circuits is the integral equation (IE) formulation combined with the method of moments (MoM) algorithm. This integral equation technique has been successfully used in the analysis of multilayered printed circuits, and it has demonstrated its accuracy and efficiency in the prediction of the electrical responses of devices without lateral walls [17], [18], as well as with shielded MMIC components [8]-[15].

The main difficulty in the integral equation formulation, however, is related to the slow convergence behavior of the modal series used to formulate the relevant spatial domain boxed Green's functions. To avoid this problem, the authors in [5] simply formulated the integral equation in the spectral domain, while in [7] spatial images are used to sum up the slowly convergent quasi-static part of the integral equation kernel. A similar approach can be found in [19], but now a second-order asymptotic term was extracted to further accelerate the convergence rate of the dynamic modal series.

The most popular technique for the series acceleration is, however, the use of the fast Fourier transform [9]-[11], but this technique restricts the MoM formulation to uniform meshes. Consequently, the discretization of arbitrary circuits of complex shapes becomes a difficult or even impossible task. Recently, two more contributions on series acceleration without the use of the fast Fourier transform have been reported [13], [14]. In [13] the modal series are accelerated by direct application of the residue theorem and contour integration techniques, while in [14] an asymptotic extraction procedure is used, combined with Poisson's summation formula and the Sommerfeld identity for the acceleration of the static part of the sums. As for the dynamic modal series they are evaluated directly without employing any further numerical transformation.

Another interesting work can be found in [20], which formulated the shielded Green's functions in the spatial domain and used the Ewalt transformation to represent the spatial images series with the complementary error function, but no dielectric layers are included in the analysis. Finally, we can mention the work in [21], which used the Shanks' transformation for the acceleration of the free-space periodic Green's function. The main problem of using the Shanks' technique, however, is that it can only accelerate 
certain types of series, so that there are combinations of source-observer point locations where the algorithm fails.

In this paper we develop two techniques that can be used for the efficient evaluation of the shielded Green's functions. The algorithms described are valid for any multilayered geometry with an arbitrary number of dielectrics, and they are applied at the Green's functions level so that any discretization scheme can be used with an integral equation technique for the analysis of complex-shaped shielded circuits and antennas. In addition, the numerically efficient evaluation of the shielded Green's functions allows us to get insight on the behavior of these Green's functions and on how the lateral walls and other geometrical parameters influence their behavior.

In Section II we review the hybrid technique based on the extraction of the quasi-static term to the spectral domain Green's functions, and we show that by extracting a second-order term the convergence of the resulting dynamic modal series can be greatly enhanced. In regard to the quasi-static terms, they are computed in the spatial domain by transforming the modal series into a fast convergent spatial images series. In this paper the resulting spatial images series are summed directly, although more elaborated techniques as in [20] can be used. A similar approach to the one presented here was used in [19], where also a second-order term was extracted to accelerate the convergence behavior of the Green's functions, in the context of the analysis of zero thickness metallic obstacles inside rectangular waveguides.

In Section III we introduce and develop a new and efficient algorithm for the computation of the shielded Green's functions. The method is directly applied to the entire Green's functions, and thus, there is no need for splitting the shielded Green's functions into quasi-static and dynamic parts. The new algorithm can be viewed as the application of the integration by parts technique to the case of discrete sequences ("sum by parts" technique), and it is shown that the computational time is reduced in several orders of magnitude with respect the first method.

In this paper, we present an exhaustive study on the convergence behavior of the techniques devised, and we include several validation examples. The paper concludes with the computation of the shielded Green's functions in several interesting situations and with the study of the influence of the lateral walls in the global Green's functions behavior. The developed algorithms are expected to find application in the analysis of complex shielded circuits and antennas using the integral equation technique.

\section{THE HYbrid APPROACH: EXTRACTION OF QUASI-STATIC TERMS}

With reference to Fig. 1, spatial domain shielded or boxed Green's functions for stratified media are usually formulated using the well-known modal expansions as in [22]

$$
G_{B}=\sum_{m=0}^{\infty} \sum_{n=0}^{\infty} \tilde{G}\left(k_{x_{m}}, k_{y_{n}}\right) f\left(k_{x_{m}}, x, x^{\prime}\right) h\left(k_{y_{n}}, y, y^{\prime}\right)
$$

where $\tilde{G}$ is the corresponding spectral domain Green's function, which for a general layered structure can be easily obtained by
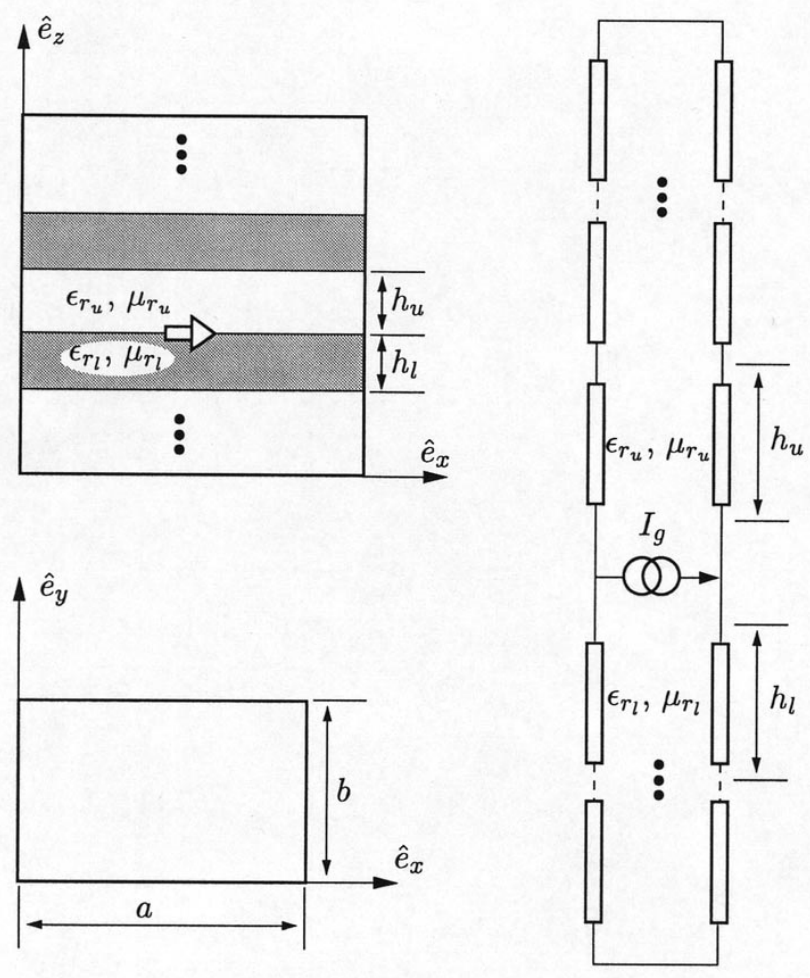

Fig. 1. Elementary dipole radiating in a multilayered shielded structure and its transverse equivalent network representation.

computing the voltages and currents in equivalent transmission line networks describing the geometry along the stratification axis (for a comprehensive review on this subject see for instance [17], [18], [23], and [24]). Moreover, $k_{x_{m}}, k_{y_{n}}$ are the transverse wave-numbers along the $\hat{e}_{x}$ and $\hat{e}_{y}$ axis, and therefore, for a rectangular waveguide they take the form

$$
k_{x_{m}}=\frac{m \pi}{a}, \quad k_{y_{n 2}}=\frac{n \pi}{b} .
$$

Finally, the functions $f$ and $h$ are combinations of sinusoidal functions whose actual form depends on the actual type of Green's functions considered. While the generic expression (1) also holds for any field component, we are here more interested in a mixed potential formulation of the problem [23], [17], [18]. Thus, Table I gives the values of $f$ and $h$ for both the magnetic vector potential and electric scalar potential boxed Green's functions, when the Sommerfeld choice is used [25]. The main practical difficulty in the evaluation of the shielded Green's functions following the formulation in (1) is related to the very slow convergence behavior of the associated infinite series. In principle, convergence properties should be investigated with respect to the two indexes $m$ and $n$. However, to simplify this cumbersome problem, we have combined both indexes into a single $k$-index, called the "influence layer" index. This principle is shown in Fig. 2 for a general series taking values of $m$ and $n$ between $-\infty$ to $+\infty$. In this case the total number of $(m, m)$ terms added as a function of the number of influence cells considered can be easily obtained as $\operatorname{Tot}(m, n)=4 k(k-1)+1$. For the series (1), the indexes $m$ and $n$ take only positive integer values, and therefore only the first quadrant in Fig. 2 should be considered. The total number of terms in this case then becomes $\operatorname{Tot}(m, n)=k^{2}$. 
TABLE I

ACTUAL Form OF THE SINUSOIDAL $f$ AND $h$ FUNCTIONS FOR ALl DiFFERENT Mixed Potential GREen's Functions COMPONENTS

\begin{tabular}{c||c|c}
\hline- & $f\left(k_{x_{m}}, x, x^{\prime}\right)$ & $h\left(k_{y_{n}}, y, y^{\prime}\right)$ \\
\hline \hline$G_{\mathrm{BA}}^{x x}$ & $\cos \left(k_{x_{m}} x\right) \cos \left(k_{x_{m}} x^{\prime}\right)$ & $\sin \left(k_{y_{n}} y\right) \sin \left(k_{y_{n}} y^{\prime}\right)$ \\
\hline$G_{\mathrm{BA}}^{y y}$ & $\sin \left(k_{x_{m}} x\right) \sin \left(k_{x_{m}} x^{\prime}\right)$ & $\cos \left(k_{y_{n}} y\right) \cos \left(k_{y_{n}} y^{\prime}\right)$ \\
\hline$G_{\mathrm{B} V}$ & $\sin \left(k_{x_{m}} x\right) \sin \left(k_{y_{n}} x^{\prime}\right)$ & $\sin \left(k_{y_{n}} y\right) \sin \left(k_{y_{n}} y^{\prime}\right)$ \\
\hline
\end{tabular}

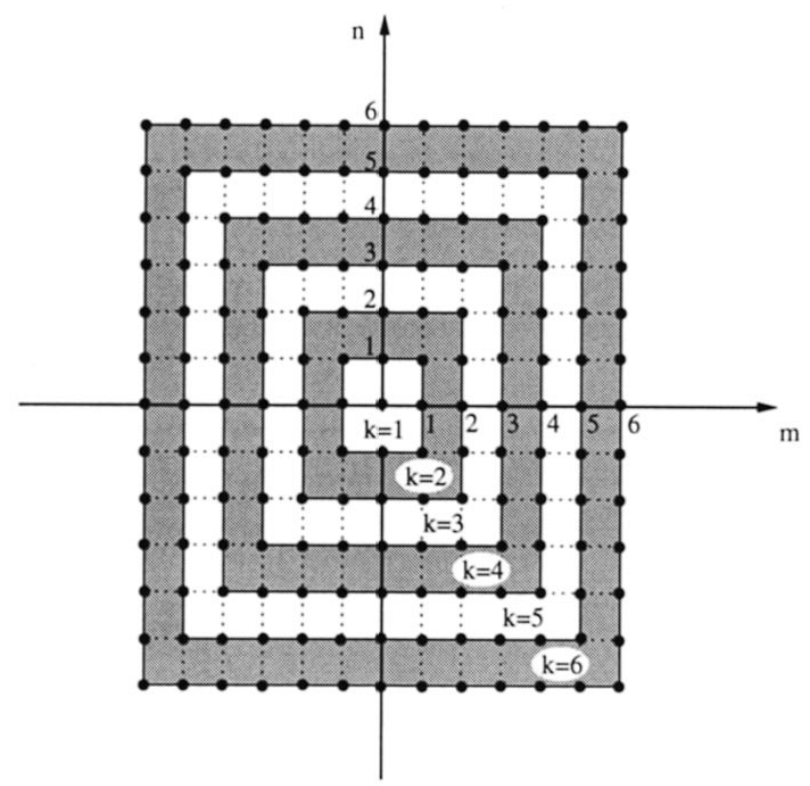

Fig. 2. Representation of a two-dimensional $(m, n)$ space by means of a one-dimensional $(k)$ space of influence layers.

As an example, we have computed the electric scalar potential in the structure of Fig. 3 with the source point placed at position $\mathrm{A}$ and the observer point placed at position $\mathrm{D}$. The convergence behavior is shown in Fig. 4 where we have plotted the absolute value of the electric scalar potential as a function of the influence cells included in the evaluation of the series. As seen, good convergence is not achieved even with $k=300$ influence cells, thus indicating a very slow convergence rate.

An alternative approach for the evaluation of the shielded Green's functions is to apply to (1) the Poisson's summation formula [22]

$$
\sum_{n=-\infty}^{\infty} G(\alpha n)=\frac{\sqrt{2 \pi}}{\alpha} \sum_{n=-\infty}^{\infty} \tilde{G}\left(\frac{2 n \pi}{\alpha}\right)
$$

with $\tilde{G}$ representing the Fourier transform of $G$. The use of the Poisson's summation formula for a two-dimensional (2-D) function together with the shifting property of the Fourier transform yields the following identity:

$$
\begin{aligned}
\sum_{m=-\infty}^{\infty} \sum_{n=-\infty}^{\infty} G(x+2 m a, y+2 n b) \\
=\frac{2}{\pi a b} \sum_{m=-\infty}^{\infty} \sum_{n=-\infty}^{\infty} \tilde{G}\left(k_{x_{m}}, k_{y_{n}}\right) \\
\quad \times \exp \left(j k_{x_{m}} x\right) \exp \left(j k_{y_{n}} y\right) .
\end{aligned}
$$

Equation (4) clearly indicates that a series involving sinusoidal functions can be transformed into a series involving shifted versions of the original function in the spatial domain. For the case of our Green's functions, the position of each elementary dipole is spatially shifted a quantity $(2 m a)$ along the $\hat{e}_{x}$ axis and $(2 n b)$ along $\hat{e}_{y}$ axis. Direct application of (4) into the Green's function in (1) leads to the following spatial images series (Fig. 5) [23]:

$$
\begin{aligned}
& G_{\mathrm{Box}}\left(x, y \mid x^{\prime}, y^{\prime}\right) \\
&= \frac{a b}{8 \pi} \sum_{m=-\infty}^{\infty} \sum_{n=-\infty}^{\infty}\left[G\left(x, y \mid+x^{\prime}+2 m a,+y^{\prime}+2 n b\right)\right. \\
&+s_{x} G\left(x, y \mid-x^{\prime}+2 m a,+y^{\prime}+2 n b\right) \\
&+s_{y} G\left(x, y \mid+x^{\prime}+2 m a,-y^{\prime}+2 n b\right) \\
&\left.+s_{x} s_{y} G\left(x, y \mid-x^{\prime}+2 m a,-y^{\prime}+2 n b\right)\right]
\end{aligned}
$$

where $G$ is the corresponding spatial domain Green's function in a layered medium of infinite transverse dimensions [26], [27], [17], [18], and $s_{x}, s_{y}$ are sign functions taking different values according to the actual type of Green's function considered. For the mixed potential Sommerfeld choice formulation, the sign functions $s_{x}$ and $s_{y}$ required to satisfy the boundary conditions for the fields at the lateral walls are collected in Table II [23]. The great advantage of using Poisson's summation formula is that the resulting spatial images series usually converge very fast. This is especially true at relatively low frequencies or when no layers are present in the structure. When frequency increases, however, surface waves might be excited and convergence is rapidly degraded.

To overcome the problem of the slow convergence rate of the series, a hybrid technique between the two described before can be attempted. Following this approach the quasi-static term of the spectral domain Green's function is first computed and then substructed to the whole spectral domain function. As it will be shown later, the modal series with the remainder thus computed converge faster, and the Poisson's summation formula is applied only to the quasi-static term. In this way the quasi-static modal series, which converges slowly (Fig. 4), is transformed into a fast convergent spatial images series. The remainder, once the quasi-static part substracted, is summed up directly in the spectral domain.

We now illustrate the procedure specifically with the electric scalar potential, being formally equivalent for any other type of Green's function. From (1) and Table I we find the explicit form of the boxed electric scalar potential Green's function as

$$
\begin{aligned}
G_{\mathrm{BV}}= & \sum_{m=0}^{\infty} \sum_{n=0}^{\infty} \tilde{G}_{V}\left(k_{x_{m}}, k_{y_{n}}\right) \sin \left(k_{x_{m}} x\right) \\
& \times \sin \left(k_{y_{n}} y\right) \sin \left(k_{x_{m}} x^{\prime}\right) \sin \left(k_{y_{n}} y^{\prime}\right) .
\end{aligned}
$$

Adding and substracting the quasi-static part of the spectral domain Green's function $G_{V_{0}}$, we obtain the following two series which must be treated separately:

$$
\begin{aligned}
G_{\mathrm{BV}}= & \sum_{m=0}^{\infty} \sum_{n=0}^{\infty}\left(\tilde{G}_{V}-G_{V_{0}}\right) \sin \left(k_{x_{m}} x\right) \sin \left(k_{y_{n}} y\right) \\
& \times \sin \left(k_{x_{m}} x^{\prime}\right) \sin \left(k_{y_{n}} y^{\prime}\right)+G_{\mathrm{BV}_{0}} \\
G_{\mathrm{BV}_{0}}= & \sum_{m=0}^{\infty} \sum_{n=0}^{\infty} G_{V_{0}}\left(k_{x_{m}}, k_{y_{n}}\right) \sin \left(k_{x_{m}} x\right) \\
& \times \sin \left(k_{y_{n}} y\right) \sin \left(k_{x_{m}} x^{\prime}\right) \sin \left(k_{y_{n}} y^{\prime}\right) .
\end{aligned}
$$




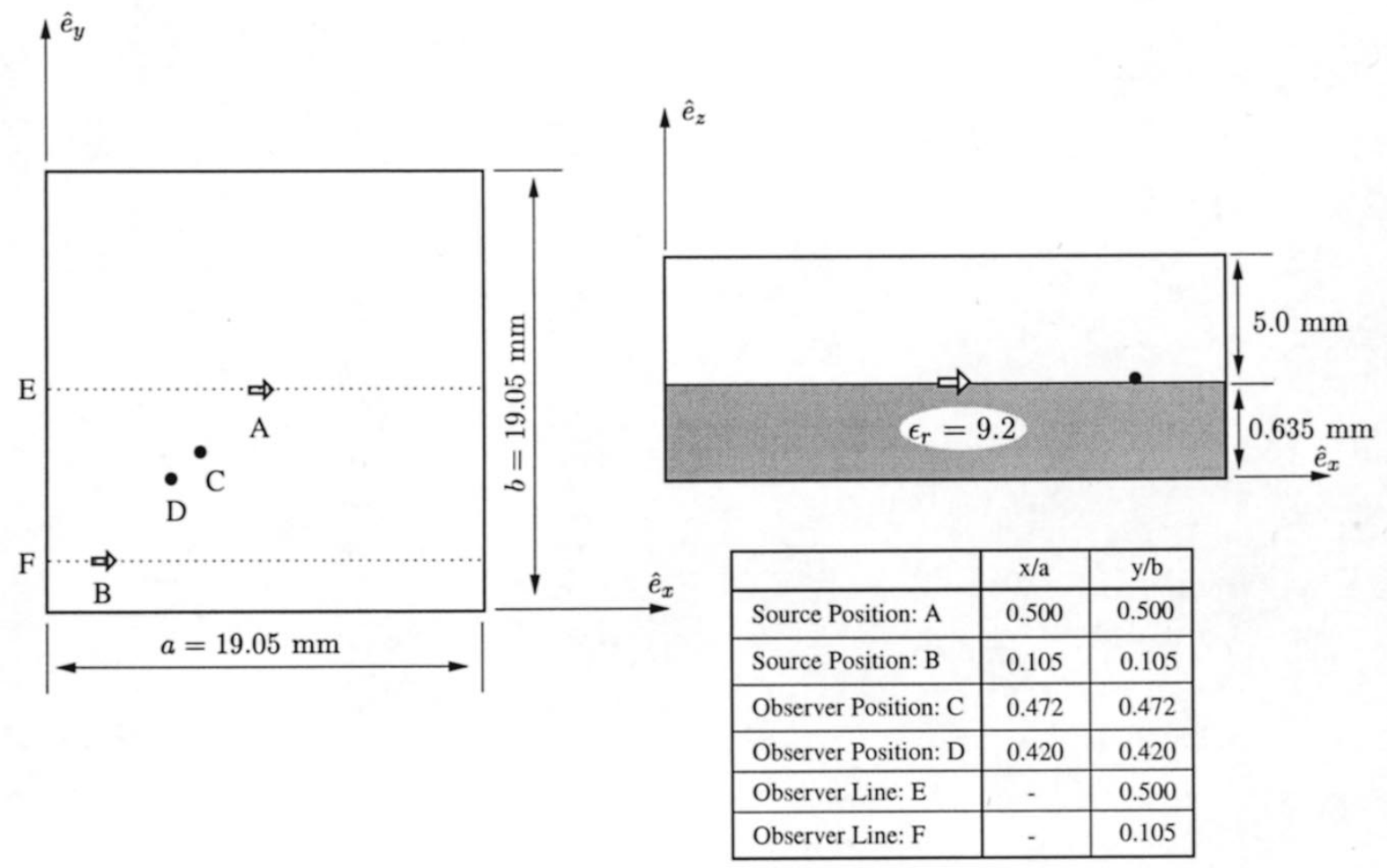

Fig. 3. Shielded microstrip structure to be studied in this paper.

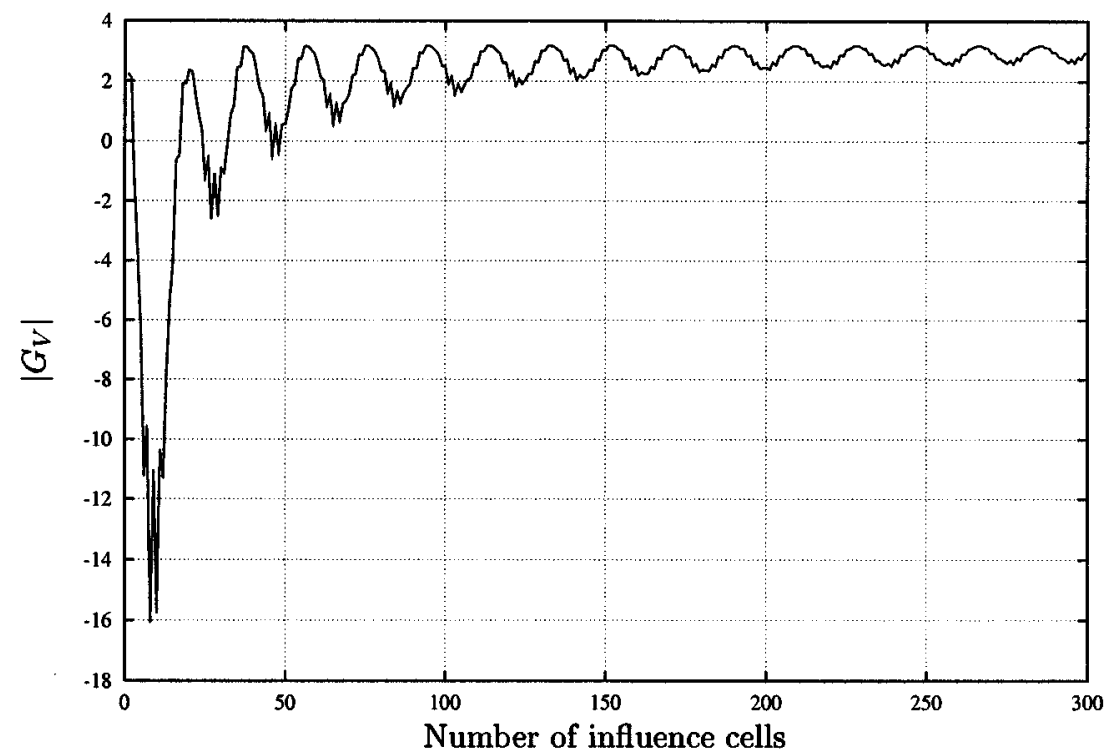

Fig. 4. Convergence behavior of the modal series associated to the electric scalar potential in the structure of Fig. 3 at a frequency of 5 GHz. Source is located at position A while observer is located at position D.

The quasi-static part of the spectral domain Green's function can now be computed by simply taking the limiting case $\underset{n \rightarrow \infty}{\substack{m \rightarrow \infty \\ n \rightarrow \infty}}$. For instance, for the case of the electric scalar potential we obtain the following quasi-static spectral domain terms:

$$
G_{V_{0}}=\frac{A^{\mathrm{TM}}}{k_{\rho_{m, n}}}+\frac{A^{\mathrm{TE}}}{k_{\rho_{m, n}}^{3}}
$$

with $A^{\mathrm{TM}}$ and $A^{\mathrm{TE}}$ being real constants obtained under TM and TE wave excitations, respectively, and the transverse or radial wavenumber has been defined in the usual way

$$
k_{\rho_{m, n}}=\sqrt{k_{x_{m}}^{2}+k_{y_{n}}^{2}} .
$$

From (8) it can be easily observed that the quasi-static series in (7b) depends on $\left(1 / k_{\rho_{m, n}}\right)$ for the TM part and on $\left(1 / k_{\rho_{m, n}}^{3}\right)$ 


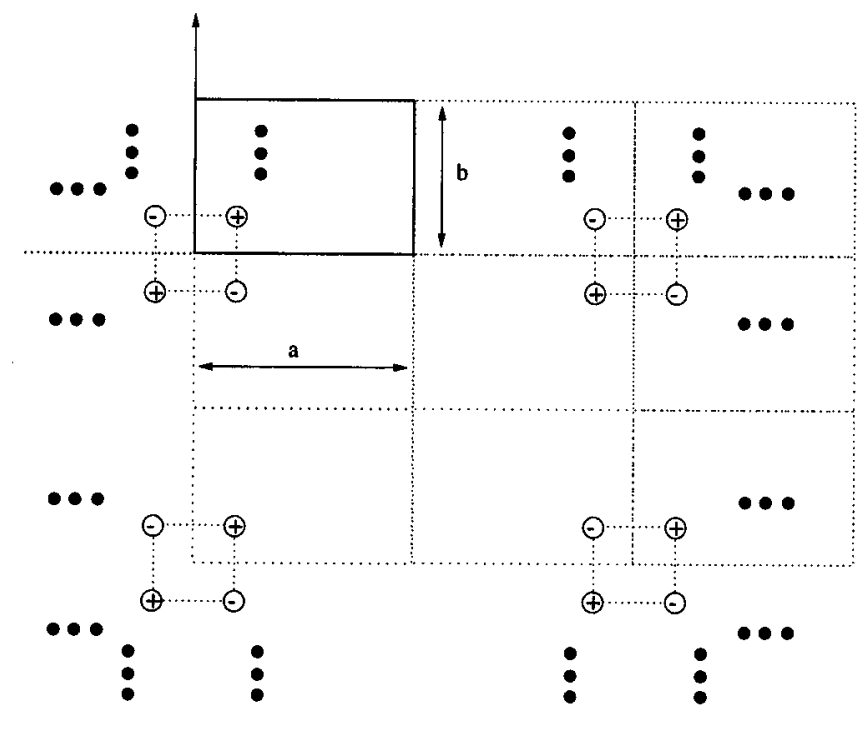

Fig. 5. Spatial images for a single unit point charge needed to satisfy boundary conditions at lateral metallic walls.

TABLE II

Value of the Sign Functions for All Different Mixed Potential GREEN'S FUNCTIONS COMPONENTS

\begin{tabular}{c||c|c}
\hline- & $s_{x}$ & $s_{y}$ \\
\hline \hline$G_{\mathrm{BA}}^{x x}$ & +1 & -1 \\
\hline$G_{\mathrm{B} A}^{y y}$ & -1 & +1 \\
\hline$G_{\mathrm{B} V}$ & -1 & -1 \\
\hline
\end{tabular}

for the TE part. In addition, the dynamic series in (7a) (once the quasi-static term is substracted) depends on $\left(1 / k_{\rho_{m, n}}^{3}\right)$ for the TM part and $\left(1 / k_{\rho_{m, n}}^{5}\right)$ for the TE part. Due to the slow convergence rate of the quasi-static TM series, it is convenient to apply the Poisson's summation formula and compute (7b) with the spatial images series in (5). To do so we first need to compute the inverse Fourier transformation of (8). For this purpose the well-known Sommerfeld integral is used, thus resulting in

$$
\begin{aligned}
G_{V_{0}}(\rho) & =\int_{0}^{\infty} J_{0}\left(k_{\rho} \rho\right) A^{\mathrm{TM}} d k_{\rho}+\int_{0}^{\infty} J_{0}\left(k_{\rho} \rho\right) \frac{A^{\mathrm{TE}}}{k_{\rho}^{2}} d k_{\rho} \\
& =\frac{A^{\mathrm{TM}}}{\rho}-A^{\mathrm{TE}} \rho
\end{aligned}
$$

which constitutes the basic spatial domain Green's function needed to construct the spatial images series is (5). As seen through out this process, the TM part of the quasi-static potential exhibiting a slow convergence behavior of $\left(1 / k_{\rho}\right)$ type in the spectral domain is transformed into a fast convergent spatial images series with $(1 / \rho)$ behavior. In addition, the TE quasi-static part of the potential in the spectral domain behaves as $\left(1 / k_{\rho}^{3}\right)$, and it is transformed into a spatial images series with behavior of $(\rho)$ type. Combining (10) with (5), the total boxed electric scalar potential Green's function in (7a) is finally written as

$$
\begin{aligned}
G_{\mathrm{BV}}= & \sum_{m=0}^{\infty} \sum_{n=0}^{\infty}\left(\tilde{G}_{V}-G_{V_{0}}\right) \sin \left(k_{x_{m}} x\right) \\
& \times \sin \left(k_{y_{n}} y\right) \sin \left(k_{x_{m}} x^{\prime}\right) \sin \left(k_{y_{n}} y^{\prime}\right)+\frac{a b}{8 \pi} \\
& \times \sum_{m=-\infty}^{\infty} \sum_{n=-\infty}^{\infty}\left[G_{V_{0}}\left(x, y \mid+x^{\prime}+2 m a,+y^{\prime}+2 n b\right)\right. \\
& -G_{V_{0}}\left(x, y \mid-x^{\prime}+2 m a,+y^{\prime}+2 n b\right) \\
& -G_{V_{0}}\left(x, y \mid+x^{\prime}+2 m a,-y^{\prime}+2 n b\right) \\
& \left.+G_{V_{0}}\left(x, y \mid-x^{\prime}+2 m a,-y^{\prime}+2 n b\right)\right] .
\end{aligned}
$$

To illustrate the convergence properties of all these series we have computed the electric scalar potential for the structure in Fig. 3 when the source is placed at position A and the observer point is located at position C. In Fig. 6 we show the relative error as a function of the influence cells added for the dynamic $\left(1 / k_{\rho}^{3}\right)$ and $\left(1 / k_{\rho}^{5}\right)$ terms of the modal series in (11). As it can be observed, by extracting a second-order quasi-static term the convergence of the dynamic modal series can be greatly enhanced.

Moreover, in Fig. 7 we show the convergence behavior of the quasi-static part after transformation into the form of a spatial images series. The results clearly indicate that the slowly convergent modal series has been transformed into a fast convergent spatial images series of $(1 / \rho)$ behavior. Also as expected, the second order quasi-static term, which is transformed into a series with $(\rho)$ behavior, exhibits a lower convergence rate, but relative errors of $0.1 \%$ can still be easily obtained.

At this point it is interesting to note that a similar procedure can be applied to the TM part of the Green's function. Following this approach, a term $\left(1 / k_{\rho}^{3}\right)$ can also be extracted from the TM part, thus resulting into a new series behaving as $\left(1 / k_{\rho}^{5}\right)$ and whose convergence behavior is shown in Fig. 6. If the remaining series with dependence $\left(1 / k_{\rho}^{3}\right)$ is transformed back to the spatial domain as shown in (10), then an additional series is obtained behaving as $(\rho)$, whose convergence properties were presented in Fig. 7.

To complete the convergence behavior study of the series developed in this section we present in Fig. 8 the number of influence cells needed in the spatial images series to achieve a numerical accuracy of $10^{-5}$ as a function of the position of the observer point. The computation is given for the frequency of 26 $\mathrm{GHz}$ and for the structure in Fig. 3, when the source is placed at two different positions (A and B). It can be seen from the figures that the smaller the distance is between source and observer points, the faster a given numerical precision is attained.

Similar results are presented in Fig. 9 but this time for a direct sum performed on the dynamic modal series. In this case the oscillating nature of the series yields to plots which are rather randomly distributed as a function of the observer point position.

Finally, the convergence behavior of the two types of series have been studied as a function of the frequency. In Table III we show the number of influence cells needed in the spatial images series to obtain different degrees of accuracy for two dif- 


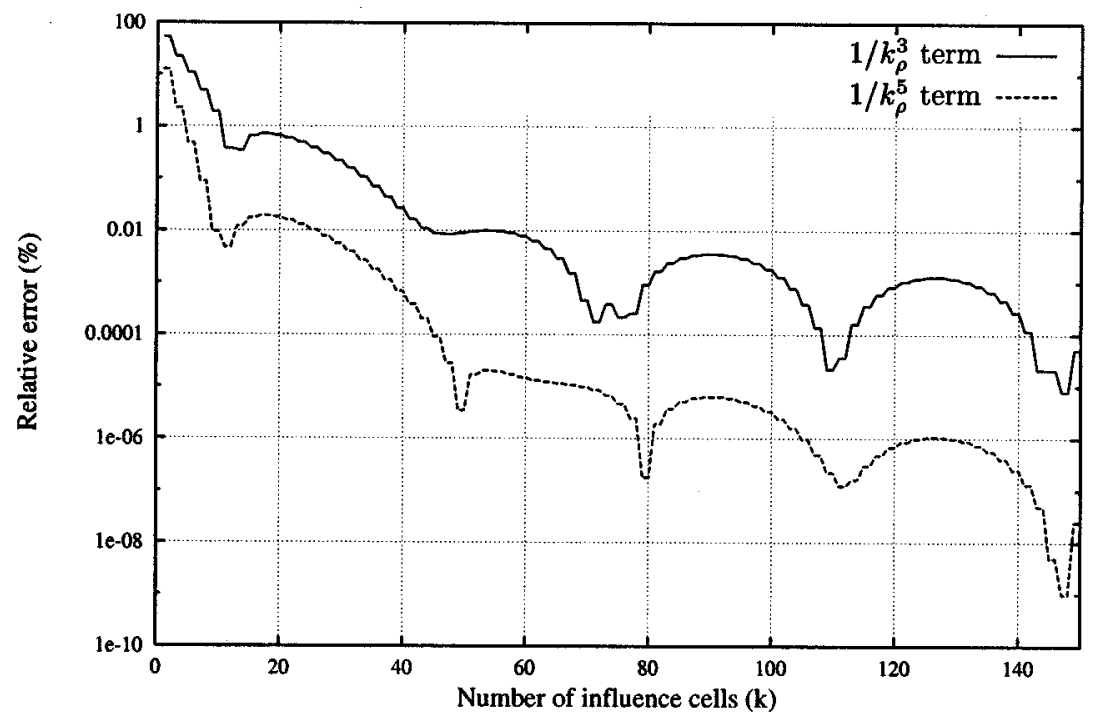

Fig. 6. Convergence of the dynamic terms $\left(1 / k_{\rho}^{3}\right)$ and $\left(1 / k_{\rho}^{5}\right)$ for the dynamic modal series in (11). Source point is placed at position A while observer point is located at position C. Frequency is $20 \mathrm{GHz}$.

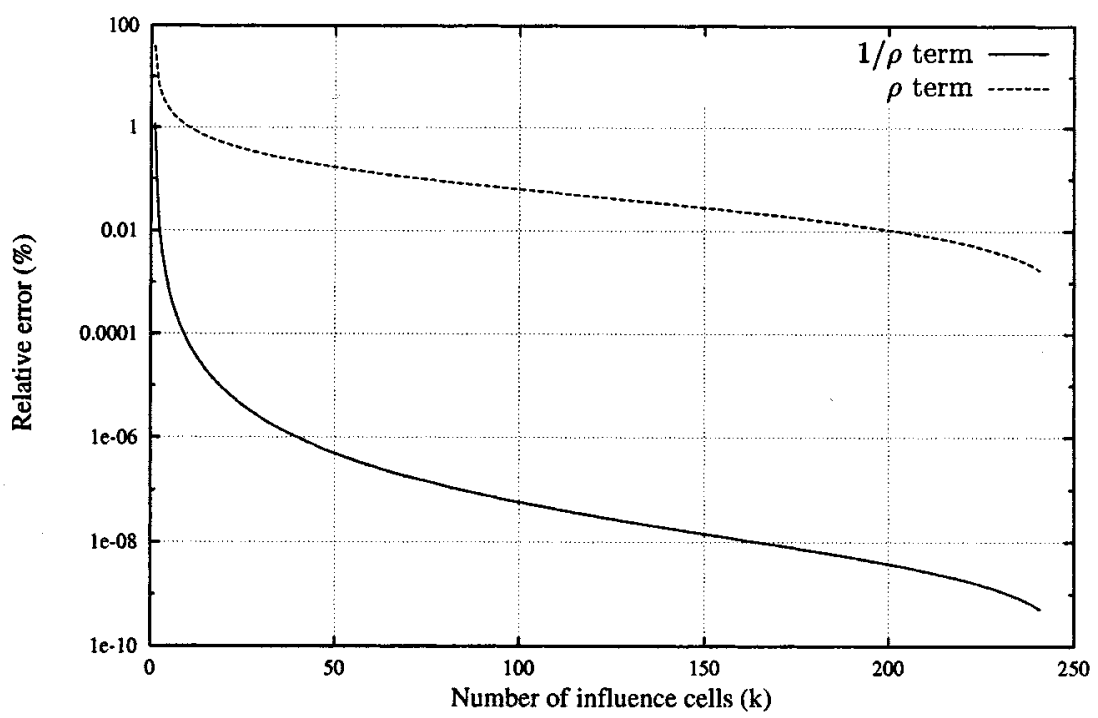

Fig. 7. Convergence of the spatial images series corresponding to a first order static term $(1 / \rho)$ and to a second-order static term $(\rho)$ for the electric scalar potential. Source point is placed at position A while observer point is located at position C. Frequency is $20 \mathrm{GHz}$.

ferent positions of the source point, when the observer point is located at position D in Fig. 3. It is interesting to mention that the data shown in Table III remains practically unchanged with frequency. A different situation is found with the convergence of the dynamic modal series. In this case the oscillating nature of the series changes with frequency, thus resulting in several critical frequencies where convergence is particularly more difficult as shown in Fig. 10.

\section{The "Summation by Parts" Technique}

In the previous section we illustrated a procedure that allows the numerical evaluation of the shielded Green's functions by extracting the quasi-static part of the spectral domain Green's functions. The modal series associated to the dynamic part, once the quasi-static part is extracted, converge fast, and direct numerical evaluation is possible. In regard to the slowly convergent quasi-static series, it is converted to the spatial domain, thus resulting in a fast convergent spatial images series. In the convergence study presented for the series developed, however, we have found several situations where convergence is difficult, and a rather large amount of influence cells need to be included to achieve an acceptable accuracy. For instance, Fig. 8 clearly indicates that the spatial images series developed might converge slowly, especially when the observer point is far from the source point. In a similar way, Figs. 9 and 10 show that there might be certain observer locations or frequencies where the convergence 

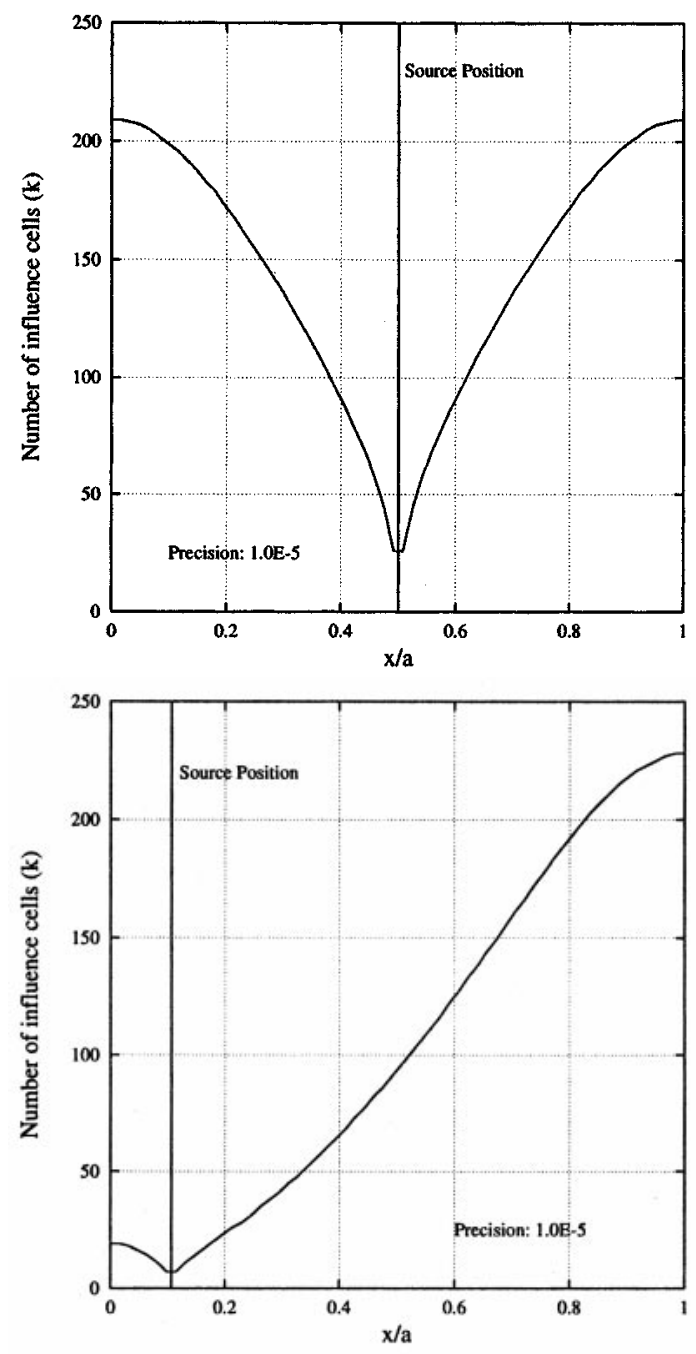

Fig. 8. Number of influence cells needed in the spatial images series to achieve given accuracy, as a function of the position of the observer point. Computation is performed for the magnetic vector potential and frequency $26 \mathrm{GHz}$.

is slower, and therefore, more influence cells need to be added to achieve the same degree of accuracy.

In this section we describe an efficient procedure for the direct evaluation of the modal series given in (1). It is shown that the technique developed greatly accelerates the convergence rate of this series so that its numerical evaluation can be effectively carried out with only few operations. The method to be described now is based on a technique for the summation of oscillating series recently developed in [28]. The algorithm described in [28] is aimed at the evaluation of the series of the following type:

$$
S_{\infty}=\sum_{n=1}^{\infty} \tilde{G}_{n} f_{n}
$$

where $\tilde{G}_{n}$ can be any general slowly variant function, and $f_{n}$ is a sinusoidal (sine or cosine) function. In [28] it was shown that an especially efficient algorithm for the numerical evaluation of the reminder associated to (12)

$$
R_{N}=\sum_{n=N}^{\infty} \tilde{G}_{n} f_{n}
$$
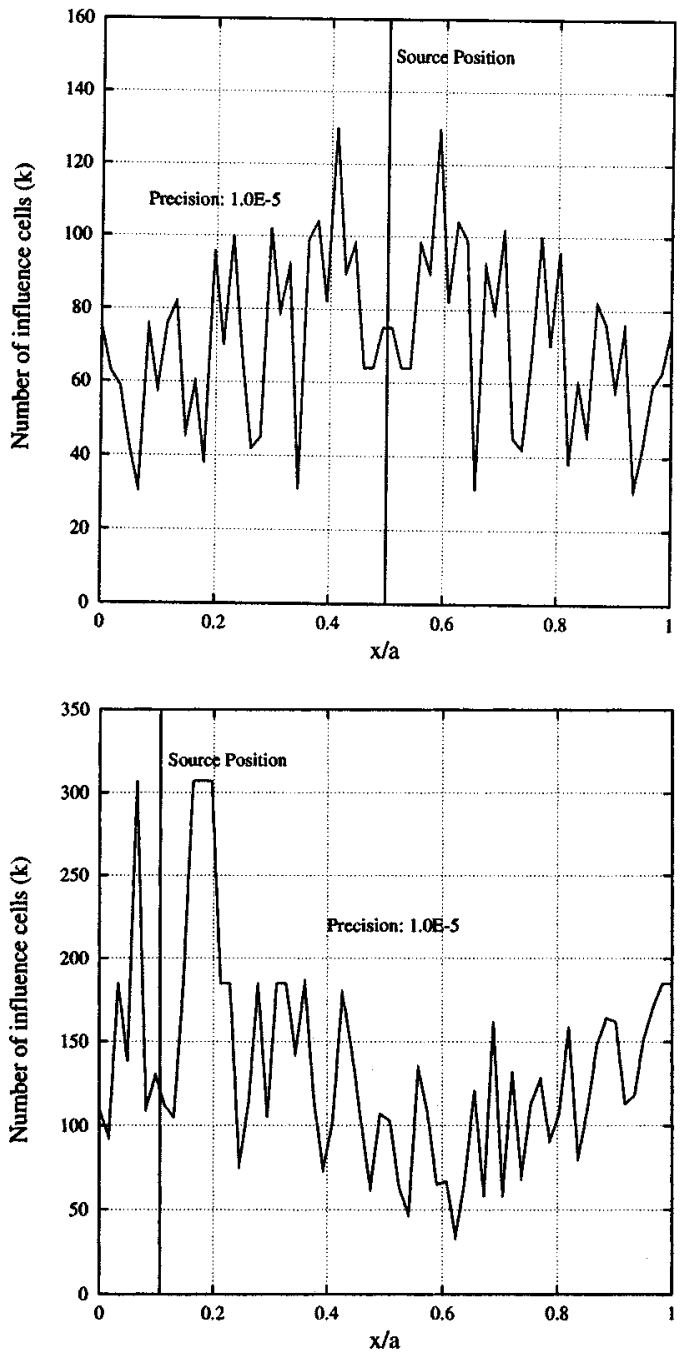

Fig. 9. Number of influence cells needed in the direct sum of the dynamic part of the electric scalar potential to achieve given accuracy, as a function of the position of the observer point. Frequency is $26 \mathrm{GHz}$.

TABLE III

Number of Influence Cells Needed in the Spatial IMAges Series to ACHIEVE DIFFERENT DEGREES OF ACCURACY. OBSERVER POINT IS

\begin{tabular}{|c|c|c|c|}
\hline & \multicolumn{2}{|c|}{ Source Coordinate } \\
\hline & & Position A & Position B \\
\hline \multirow{3}{*}{ 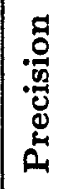 } & $10^{-3}$ & 11 & 14 \\
\hline & $10^{-4}$ & 30 & 41 \\
\hline & $10^{-5}$ & 90 & 126 \\
\hline
\end{tabular}
LOCATED AT POSITION D

is to use the following transformation:

$$
R_{N}=\sum_{n=N}^{\infty} \tilde{G}_{n}^{(-1)} f_{n}^{(+1)}=\sum_{i=1}^{\infty} \tilde{G}_{N}^{(-i)} f_{N-1}^{(i+1)}
$$



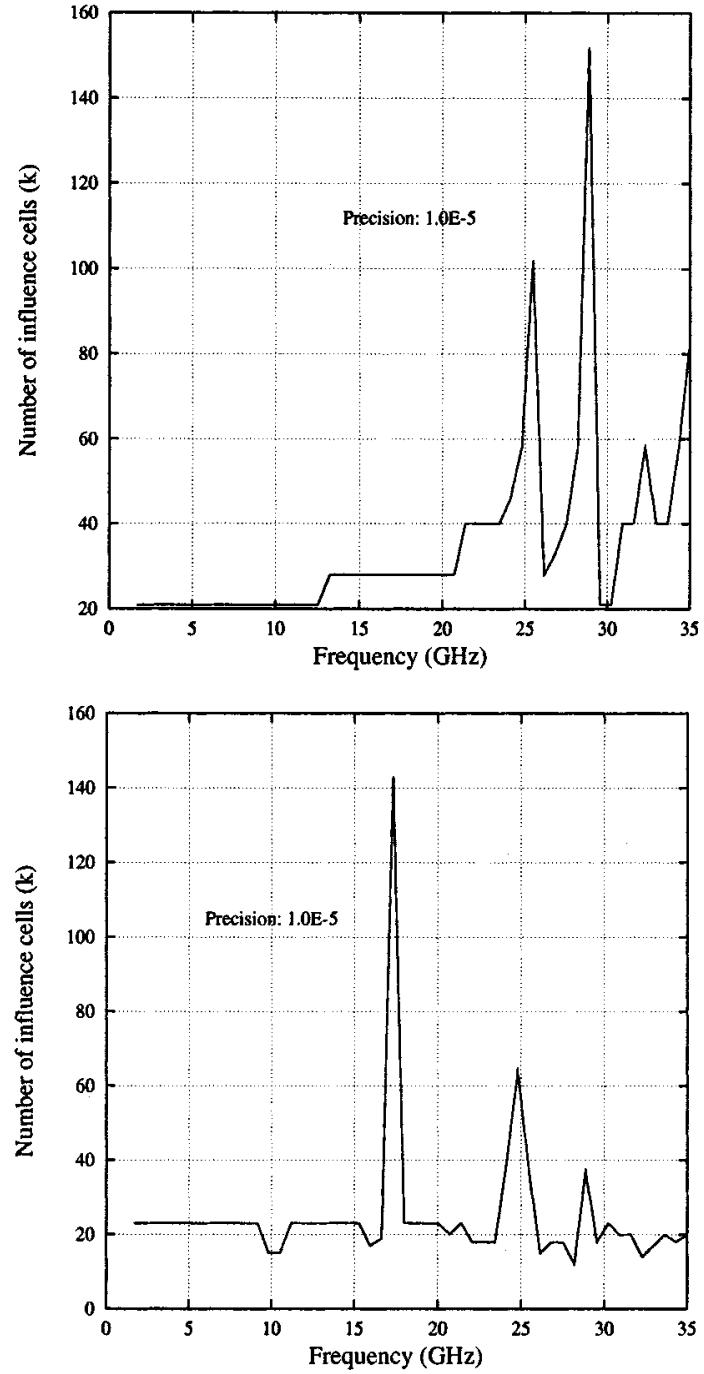

Fig. 10. Number of influence cells in the direct sum of the dynamic part of the electric scalar potential needed to achieve given accuracy, as a function of the frequency.

where $f_{n}^{(+i)}$ are higher order sums and $\tilde{G}_{n}^{(-i)}$ higher order differences defined as

$$
\begin{aligned}
& \tilde{G}_{n}^{(-1)}=\tilde{G}, \quad \tilde{G}_{n}^{(-i)}= \tilde{G}_{n+1}^{(-i+1)}-\tilde{G}_{n}^{(-i+1)}, \\
& \text { for all } i=2,3,4 \ldots, \\
& f_{n}^{(+1)}=f_{n}, \quad f_{n}^{(+i)}=\sum_{\substack{k=n+1 \\
\text { for all } i=2,3,4 \ldots}}^{\infty} f_{k}^{(i-1)},
\end{aligned}
$$

From above equations we can easily see that the algorithm can be interpreted as the successive application of an integration by parts procedure to the original discrete sequence ("summation by parts" technique) [28].

This technique, developed for one-dimensional (1-D) oscillating series, can be easily extended to the case of a 2-D series as the one shown in (1). First we define the related 2-D reminder as

$$
R_{M, N}=\sum_{m=M}^{\infty} \sum_{n=N}^{\infty} \tilde{G}_{m, n} f_{m} h_{n}
$$
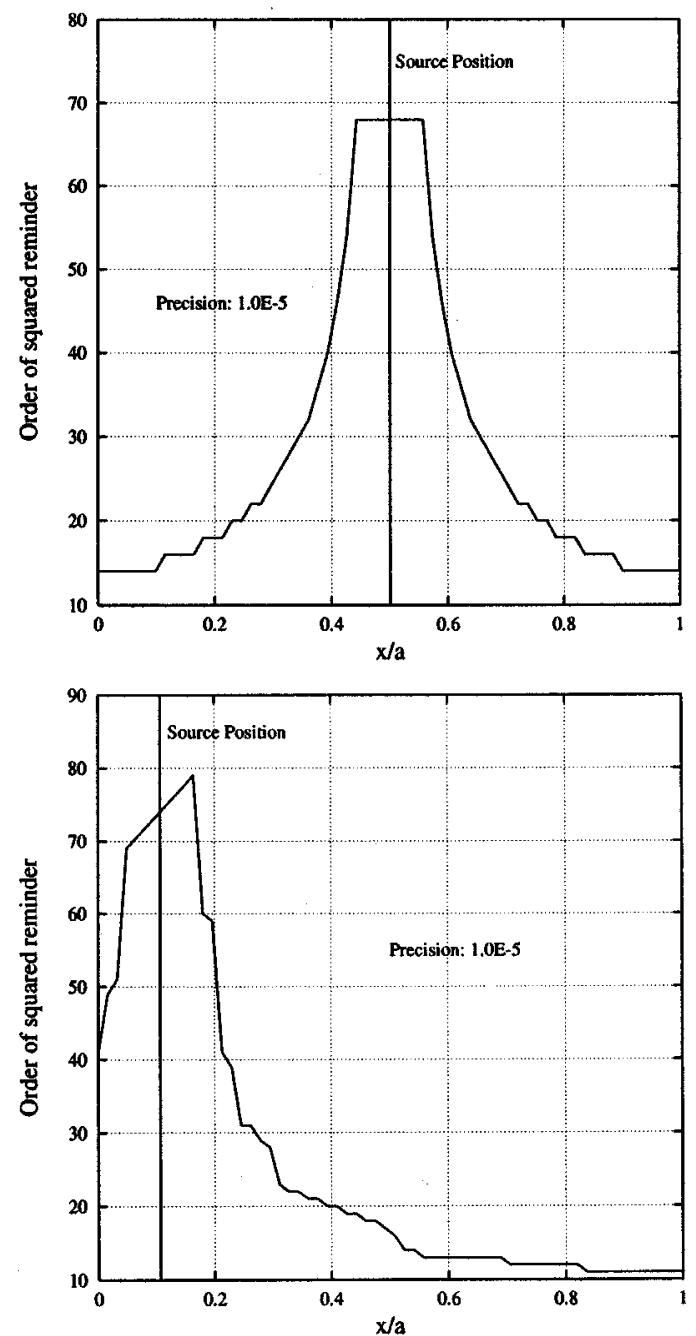

Fig. 11. Number of iterations needed in the new algorithm to achieve given accuracy, as a function of the position of the observer point. Frequency is 26 GHz.

\begin{tabular}{|c|c|c|c|}
\hline & \multicolumn{2}{|c|}{ Source Coordinate } \\
\hline & & Position A & Position B \\
\hline \multirow{3}{*}{ 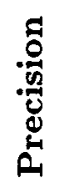 } & $10^{-3}$ & 32 & 11 \\
\hline & $10^{-4}$ & 34 & 16 \\
\hline & $10^{-5}$ & 46 & 18 \\
\hline
\end{tabular}

TABLE IV

Order of THE SQuared Reminder NEEDED IN THE NEW Algorithm to ACHIEVE DIFFERENT DEGREES OF ACCURACY. OBSERVER POINT IS LOCATED AT POSITION D

where $\tilde{G}_{m, n}$ represents the spectral domain Green's function in a real problem, and $f_{m}, h_{n}$ are simple products of sinusoidal functions as shown in Table I. To proceed, first we introduce the following notation:

$$
\begin{aligned}
\tilde{G}_{m, n}^{(-1,-1)} & =\tilde{G}_{m, n}, \quad f_{m}^{(1)}=f_{m} \\
h_{n}^{(1)} & =h_{n}
\end{aligned}
$$


so that (16) is written as

$$
R_{M, N}=\sum_{m=M}^{\infty} f_{m}^{(1)} \sum_{n=N}^{\infty} \tilde{G}_{m, n}^{(-1,-1)} h_{n}^{(1)} .
$$

In this last equation we can define the following partial 1-D sum:

$$
\Psi(m, N)=\sum_{n=N}^{\infty} \tilde{G}_{m, n}^{(-1,-1)} h_{n}^{(1)}
$$

so that the reminder in (18) becomes

$$
R_{M, N}=\sum_{m=M}^{\infty} \Psi(m, N) f_{m}^{(1)} .
$$

The problem is then reduced to the evaluation of several 1-D sequences as shown in (19) and (20). In fact, we need to evaluate $\Psi(m, N)$ for several values of the index $m$, so that (19) requires the evaluation of several 1-D sequences. Once $\Psi(m, N)$ is computed, we just use it to evaluate the additional 1-D sequence given in (20). Using now on (19) the 1-D summation by parts algorithm shown in (14), we obtain

$$
\Psi(m, N)=\sum_{k=1}^{\infty} \tilde{G}_{m, N}^{(-1,-k)} h_{N-1}^{(k+1)}
$$

where in a similar way as in (15), the difference and sum functions with respect the inner index $n$ have been defined as

$$
\begin{aligned}
\tilde{G}_{m, n}^{(-1,-k)} & =\tilde{G}_{m, n+1}^{(-1,-k+1)}-\tilde{G}_{m, n}^{(-1,-k+1)} \\
h_{n}^{(k)} & =\sum_{i=n+1}^{\infty} h_{i}^{(k-1)}, \quad \text { for all } k=2,3,4, \ldots
\end{aligned}
$$

We can now introduce (21) into (20) to write

$$
R_{M, N}=\sum_{m=M}^{\infty} \sum_{k=1}^{\infty} \tilde{G}_{m, N}^{(-1,-k)} h_{N-1}^{(k+1)} f_{m}^{(1)}
$$

and if the order of summations are interchanged

$$
R_{M, N}=\sum_{k=1}^{\infty} h_{N-1}^{(k+1)} \sum_{m=M}^{\infty} \tilde{G}_{m, N}^{(-1,-k)} f_{m}^{(1)} .
$$

Now we apply again the 1-D summation by parts algorithm to the resulting inner sum in (24), therefore obtaining

$$
R_{M, N}=\sum_{i=1}^{\infty} \sum_{k=1}^{\infty} \tilde{G}_{M, N}^{(-i,-k)} f_{M-1}^{(i+1)} h_{N-1}^{(k+1)}
$$

where the new difference and sum functions with respect the outer $m$ index have been defined as

$$
\begin{aligned}
& \tilde{G}_{m, n}^{(-i,-k)}=\tilde{G}_{m+1, n}^{(-i+1,-k)}-\tilde{G}_{m, n}^{(-i+1,-k)} \\
& f_{n}^{(i)}=\sum_{k=n+1}^{\infty} f_{k}^{(i-1)} \\
& \quad \text { for all }\left\{\begin{array}{l}
i=1,2,3, \ldots, \quad i=k \neq 1 . \\
k=1,2,3, \ldots,
\end{array}\right.
\end{aligned}
$$

Equation (25) represents the basic transformation allowing us to efficiently compute a 2-D reminder using the sum by parts algorithm, and as it can be seen it involves only the successive difference functions $\widetilde{G}_{m, n}^{(-i,-k)}$ and the successive sum functions $f_{m}^{(i)}$ and $h_{n}^{(k)}$. While the successive difference functions can be easily evaluated numerically, explicit analytical expressions must be obtained for the successive sum functions. For the computation of the Green's functions given in (1) and in Table I, it is most convenient to reduce all sine and cosine products into the sum of basic single cosine functions. Following this approach, the elementary sinusoidal functions $f_{m}$ and $h_{n}$ of (16) take the form of simple cosine functions, and therefore, the successive sum functions given in (22) and (26) can be easily obtained analytically by applying simple geometrical progression summation formula. The recurrent relation yields to the following general expression for both $f_{m}^{(i)}$ and $h_{n}^{(k)}$ successive sum functions:

$$
\begin{gathered}
f_{n}^{(1)}=h_{n}^{(1)}=\cos (n x) \\
f_{n}^{(i)}=h_{n}^{(i)}=\frac{\cos \left\{(i-1) \frac{\pi}{2}+\left[n+(i-1) \frac{1}{2}\right] x\right\}}{2^{(i-1)}\left[\sin \left(\frac{x}{2}\right)\right]^{(i-1)}} \\
\text { for all } i=2,3,4 \ldots
\end{gathered}
$$

Using the analytical definition in (27b), (25) can be applied to all basic cosine series in which the whole Green's functions are decomposed. The final Green's functions are obtained later by combining all basic cosine series appropriately.

To show the effectiveness of the approach developed, we have computed the relative error obtained during the numerical computation of the reminder according to (25), as a function of the number of iterations used in the inner (index $k$ ) and outer (index i) sum by parts indexes. The computation was performed in the structure of Fig. 3 when the source is placed at position $\mathrm{B}$, the observer point is located at position $\mathrm{C}$, and the frequency is 20 GHz. In this computation the order of the reminder was taken $M=40, N=40$, and we found that only four iterations do suffice in both indexes to achieve impressive numerical precision of the order of $10^{-5}$.

The convergence properties of the new algorithm have also been studied as a function of the source and observer positions and as a function of the frequency. In Fig. 11 we show the order of the squared reminder $(M=N)$ needed to achieve numerical precision of $10^{-5}$ as a function of the position of the observer point, and for two different locations of the source (position A and B). For this computation the number of summation by parts iterations have been fixed to $i=k=6$, and as we observe close to the singularity the order of the reminder needs to be slightly increased to keep the required numerical precision.

Furthermore, in Table IV we show the same quantity needed to achieve different degrees of accuracy when the observer point is placed at position $\mathrm{D}$ and for two different locations of the source point (A and B). As seen, in any case the required precision is attained fast; moreover, these results turn out to be essentially independent on frequency.

\section{RESULTS}

Based on the theory described in this paper a computer code has been developed for the evaluation of the mixed potential shielded Green's functions in a multilayered environment. For validation purposes we have computed the mixed potential Green's functions for the structure in Fig. 3 with the two techniques, and for different combinations of source-observer 


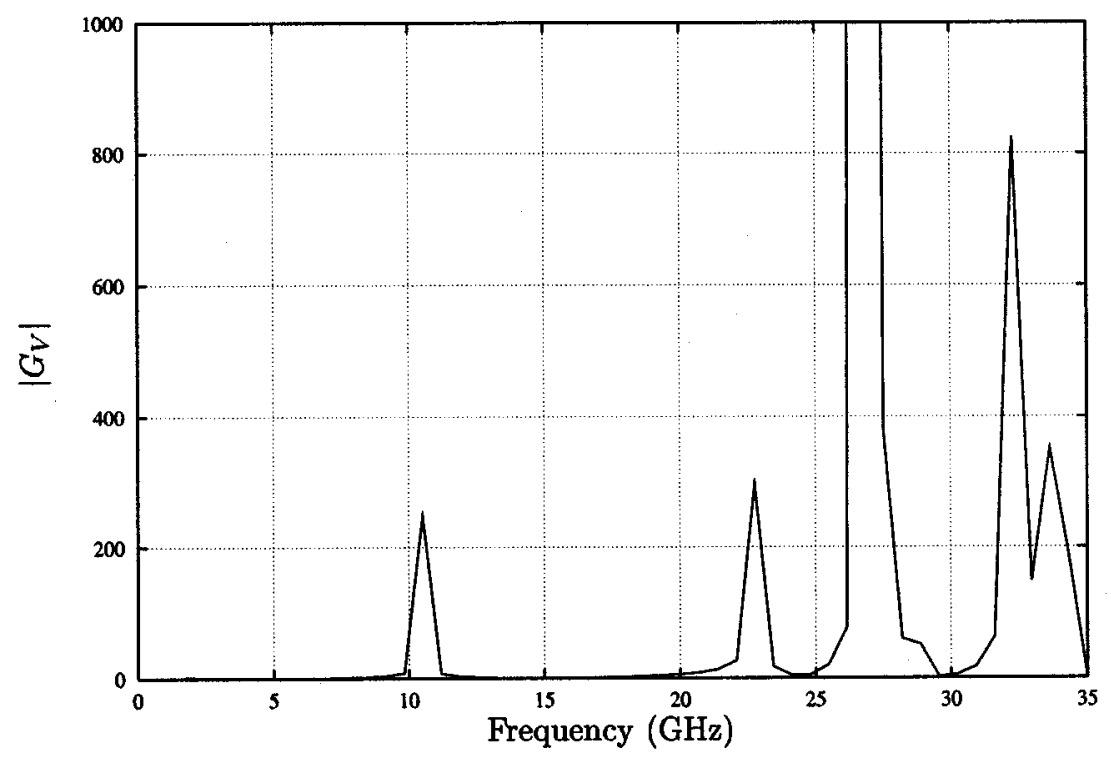

Fig. 12. Electric scalar potential versus frequency. Source point located at position A. Observer point located at position D.

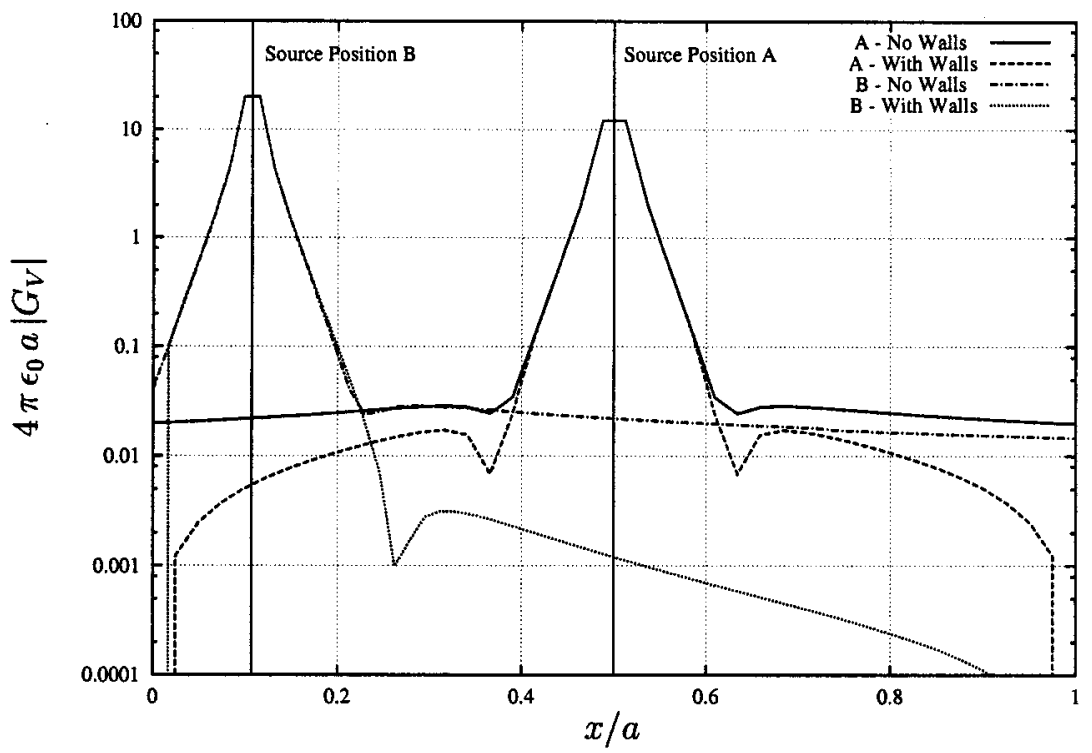

Fig. 13. Open and shielded electric scalar potential Green's functions for the structure shown in Fig. 3 at a frequency of 4 GHz. Source is located at positions A and B. Observer is located along lines $\mathrm{E}$ and $\mathrm{F}$.

points and frequencies, always finding an agreement better than $10^{-6}$.

Once the technique has been validated and its convergence properties extensively studied, we have investigated the effects and influence of the lateral walls in the electric scalar potential Green's function of the structure in Fig. 3. First we have computed the electric scalar potential as a function of the frequency when the source is located at position A and the observer point at position D. The results are shown in Fig. 12, and as we observe there are frequencies where the electric scalar potential increases abruptly corresponding clearly to the natural resonances of the box.

In Fig. 13 we show the electric scalar potential with and without lateral walls when the source is placed at two different locations ( $\mathrm{A}$ and $\mathrm{B}$ ) and at a frequency of $4 \mathrm{GHz}$, corresponding to a frequency far below the first resonance of the box. We can see that at this frequency the potential rapidly decreases when the observer point is far from the source point and that close to the singularity the effect of the lateral walls is negligible since the potentials with and without the lateral walls are essentially the same.

Similar results are presented in Fig. 14, but this time at a frequency of $15 \mathrm{GHz}$, corresponding to a frequency between the first and second resonance of the box. It can be observed that the potential with lateral walls starts exhibiting oscillations, thus indicating the existence of a standing wave due to the presence of the walls. The same comparison is finally presented in Fig. 15 but for the frequency of $24 \mathrm{GHz}$, corresponding to a frequency 


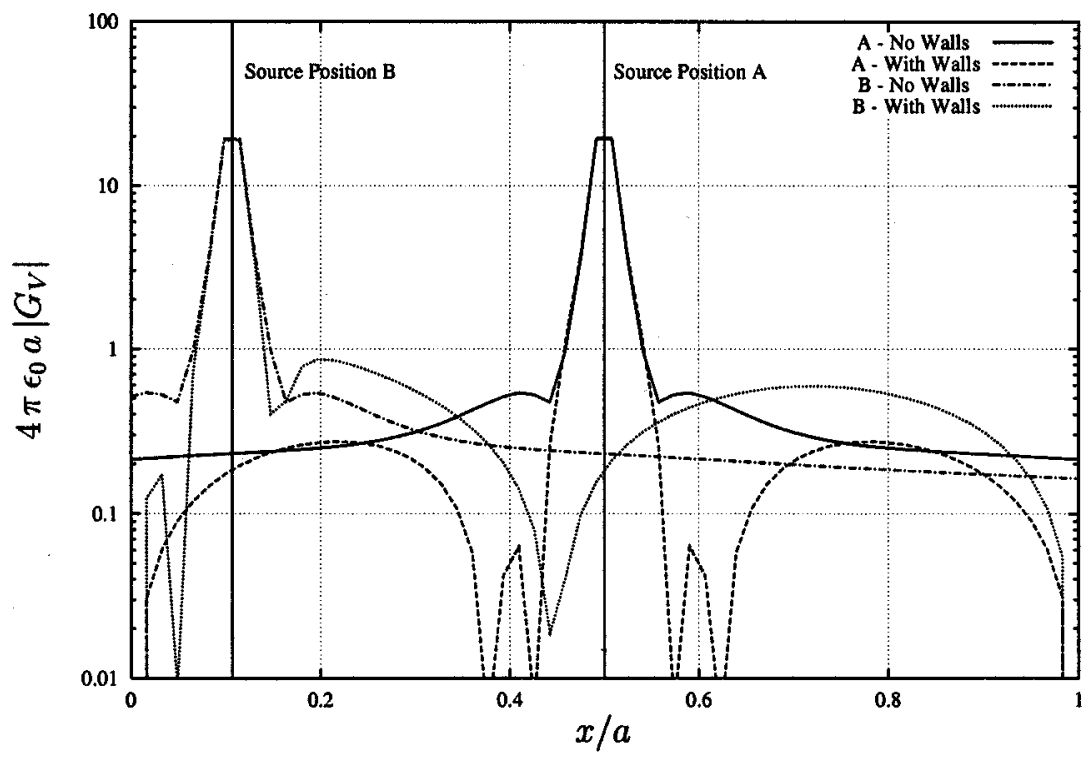

Fig. 14. Open and shielded electric scalar potential Green's functions for the structure shown in Fig. 3 at a frequency of 15 GHz. Source is located at positions $\mathrm{A}$ and $\mathrm{B}$. Observer is located along lines $\mathrm{E}$ and $\mathrm{F}$.

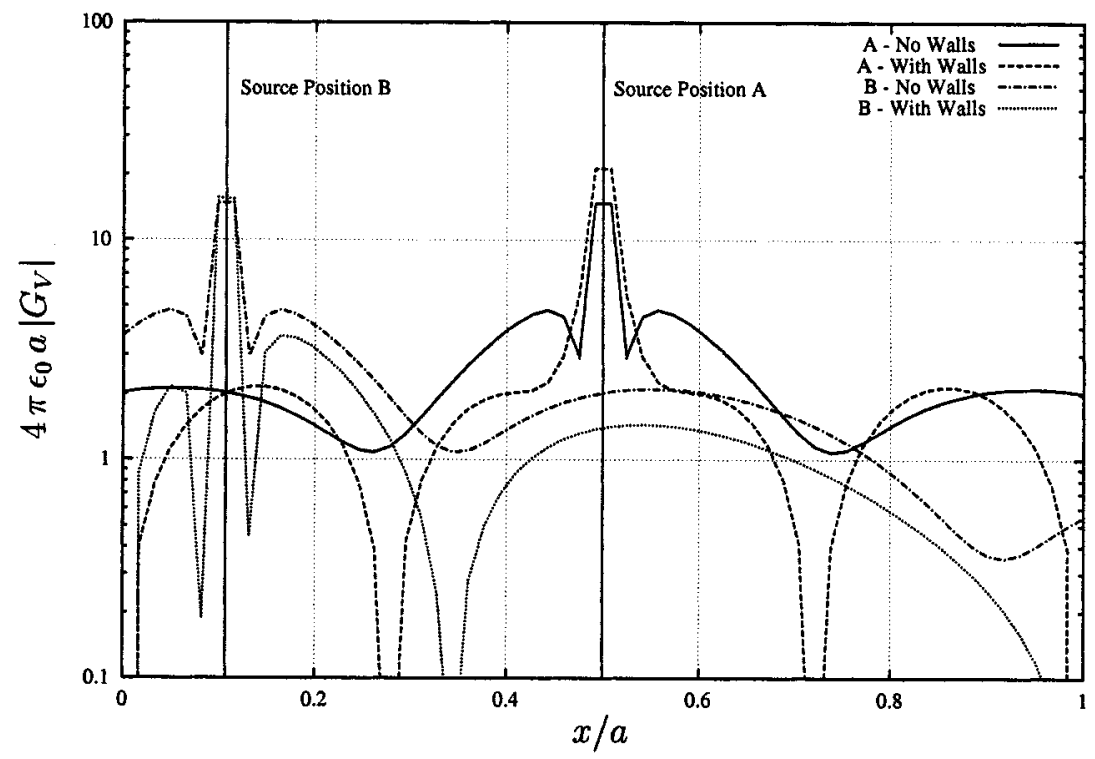

Fig. 15. Open and shielded electric scalar potential Green's functions for the structure shown in Fig. 3 at a frequency of 24 GHz. Source is located at positions $\mathrm{A}$ and $\mathrm{B}$. Observer is located along lines $\mathrm{E}$ and $\mathrm{F}$.

between the second and third resonance. In this case the potential computed without lateral walls also exhibits oscillations, but the boundary conditions at the lateral walls are only satisfied by the shielded potential Green's function.

Finally, it would be interesting to study the form of the potential at the resonance frequencies of the box. For this purpose we have computed the electric scalar potential at the frequency of $10.49 \mathrm{GHz}$ corresponding to the first resonance of the box as shown in Fig. 12. The results are shown in Fig. 16, and it can be observed that the absolute value of the potential suddenly in- creases, taking essentially the form of the dominant mode propagating in the waveguide. Also, it is interesting to point out that the potential without lateral walls does not experience any resonance effect, and therefore, at this frequency both potentials (with and without lateral walls) are very different.

It is important to mention that the same results as those presented above can also be obtained with the quasi-static term extraction technique, and no visual difference can be appreciated in the graphics. However, the reduction in computational time is dramatic when the new algorithm is used. As 


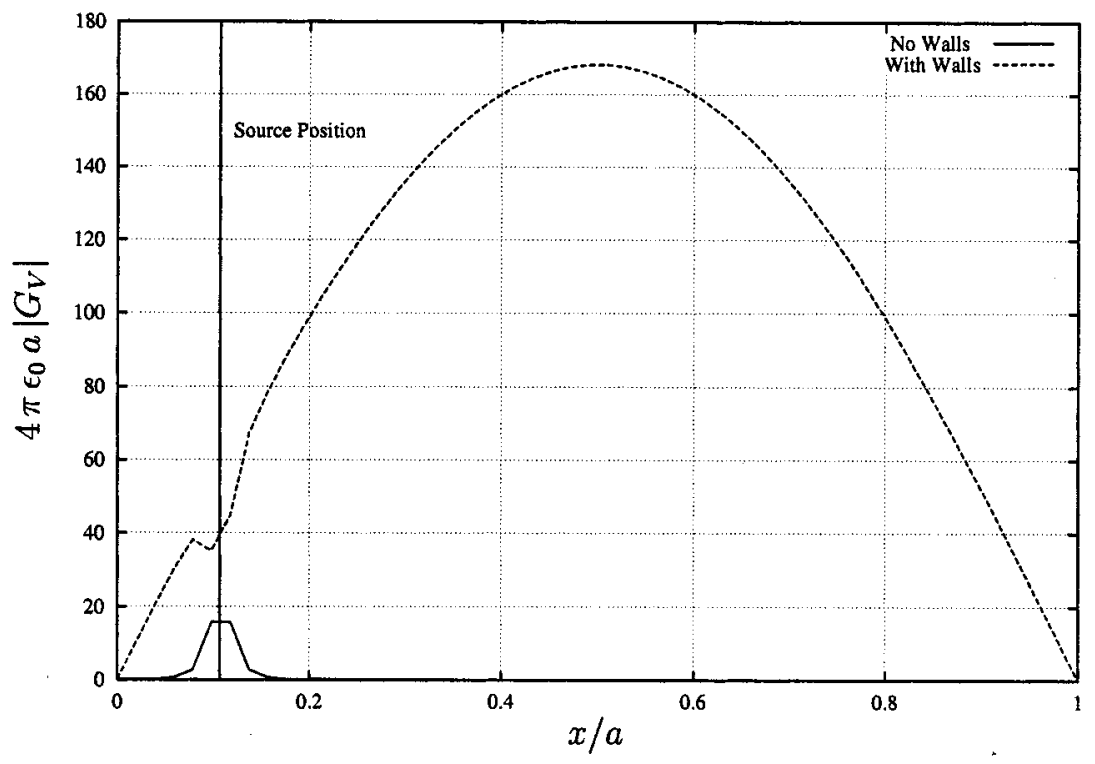

Fig. 16. Electric scalar potential at Frequency $10.49 \mathrm{GHz}$ corresponding to first resonance in Fig. 12. Source point placed at position B. Observer point located at line $\mathrm{F}$.

an example, the quasi-static term extraction technique takes $1 \mathrm{~min} 56 \mathrm{~s}$ for the computation of 60 points in Fig. 15, and only $16 \mathrm{~s}$ when the newly developed algorithm is used, on an $\mathrm{HP} 712 / 80$ platform.

\section{CONCLUSION}

In this paper we have presented two techniques allowing the computation of the mixed potential Green's functions when source and observer points are enclosed in a metallic cavity. The first technique is based on the extraction of the quasi-static term to the global spectral domain Green's function. The resulting dynamic part, once the quasi-static term is extracted, converges faster and direct evaluation is possible. In regard to the quasi-static part, it is transformed into the spatial domain, thus resulting into fast convergent spatial images series with $(1 / \rho)$ dependence. Numerical results have effectively shown that convergence of the dynamic modal series can be greatly improved if a second-order quasi-static term is extracted from the spectral domain Green's functions. Following this approach, however, care must be exercised in the summation of the additional spatial images series which now have only a ( $\rho$ ) dependence.

To accelerate the computation of the shielded Green's functions a new and efficient procedure has been presented, which can be viewed as the application of the integration by parts algorithm to discrete sequences. The algorithm is directly applied to the computation of the shielded Green's functions, greatly enhancing the convergence properties of the modal series involved. The result is that the whole shielded Green's functions are obtained in a single block of calculations (no need to extract quasi-static terms) and with only few numerical operations. It has been observed that the gain in computational time with respect traditional techniques is considerable.

\section{REFERENCES}

[1] J. C. Cheng, N. I. Dib, and L. P. B. Katehi, "Theoretical modeling of cavity-backed patch antennas using a hybrid technique," IEEE Trans. Antennas Propagat., vol. 43, pp. 1003-1013, Sept. 1995.

[2] J. M. Jin and J. L. Volakis, "A hybrid finite element method for scattering and radiation by microstrip patch antennas and arrays residing in a cavity," IEEE Trans. Antennas Propagat., vol. 39, pp. 1598-1604, Nov. 1991.

[3] — , "Scattering and radiation analysis of three-dimensional cavity arrays via a hybrid finite-element method," IEEE Trans. Antennas Propagat., vol. 41, pp. 1580-1586, Nov. 1993.

[4] J. Gong, J. L. Volakis, A. C. Woo, and H. T. G. Wang, "A hybrid finite element-boundary integral method for the analysis of cavity-backed antennas of arbitrary shape," IEEE Trans. Antennas Propagat., vol. 42, pp. 1233-1242, Sept. 1994.

[5] J. Y. Lee, T. S. Horng, and N. G. Alexopoulos, "Analysis of cavitybacked aperture antennas with a dielectric overlay," IEEE Trans. Antennas Propagat., vol. 42, pp. 1556-1562, Nov. 1994.

[6] F. Zavosh and J. T. Aberle, "Infinite phased arrays of cavity-backed patches," IEEE Trans. Antennas Propagat., vol. 42, pp. 390-398, Mar. 1994.

[7] G. G. Gentili, L. E. Garcia-Castillo, M. S. Palma, and F. Perez-Martinez, "Green's function analysis of single and stacked rectangular microstrip patch antennas enclosed in a cavity," IEEE Trans. Antennas Propagat., vol. 45, pp. 573-579, Apr. 1997.

[8] L. P. Dunleavy and P. B. Katehi, "A generalized method of analyzing shielded thin microstrip discontinuities," IEEE Trans. Microwave Theory Tech., vol. 36, pp. 1758-1766, Dec. 1988.

[9] J. C. Rautio and R. F. Harrington, "An electromagnetic time-harmonic analysis of shielded microstrip circuits," IEEE Trans. Microwave Theory Tech., vol. 35, pp. 726-730, Nov. 1987.

[10] C. J. Railton and S. A. Meade, "Fast rigorous analysis of shielded planar filters," IEEE Trans. Microwave Theory Tech., vol. 40, pp. 978-985, May 1992.

[11] A. Hill and V. K. Tripathi, "An efficient algorithm for the three-dimensional analysis of passive microstrip components and discontinuities for microwave and millimeter-wave integrated circuits," IEEE Trans. Microwave Theory Tech., vol. 39, pp. 83-91, Jan. 1991.

[12] R. Faraji-Dana and Y. L. Chow, "Accurate and efficient CAD tool for the design of optimum packaging for (M)MICs," Proc. IEE Microwave Antennas and Propagation, vol. 142, pp. 81-88, Apr. 1995.

[13] S. Hashemi-Yeganeh, "On the summation of double infinite series field computations inside rectangular cavities," IEEE Trans. Microwave Theory Tech., vol. 43, pp. 641-646, Mar. 1995. 
[14] G. V. Eleftheriades, J. R. Mosig, and M. Guglielmi, "A fast integral equation technique for shielded planar circuits defined on nonuniform meshes," IEEE Trans. Microwave Theory Tech., vol. 44, pp. 2293-2296, Dec. 1996.

[15] L. P. Dunleavy and P. B. Katehi, "Shielding effects in microstrip discontinuities," IEEE Trans. Microwave Theory Tech., vol. 36, pp. 1767-1774, Dec. 1988.

[16] A. Alvarez-Melcon and J. R. Mosig, "A Software Tool for the Analysis and Design of the Conformal Array Single Elementary Radiator," LEMA, Swiss Federal Inst. Technol., Lausanne, Switzerland, Tech. Rep. LEMA-RTAA-97-01, ESTEC/Contract 11 698/95/NL/SB, June 1997.

[17] M.-J. Tsai, F. D. Flaviis, O. Fordham, and N. G. Alexopoulos, "Modeling planar arbitrarily shaped microstrip elements in multilayered media," IEEE Trans. Microwave Theory Tech., vol. 45, pp. 330-337, Mar. 1997.

[18] M.-J. Tsai, C. Chen, and N. G. Alexopoulos, "Sommerfeld integrals in modeling interconnects and microstrip elements in multi-layered media," Electromagnetics, vol. 18, no. 3, pp. 267-288, 1998. Special Issue-The Centennial of Sommerfeld's Diffraction Problem.

[19] G. G. Gentili and G. de Angelis, "Efficient analysis of scattering by arbitrarily shaped thin obstacles in rectangular waveguide," J. Electromagnetic Waves Applicat., vol. 12, no. 1, pp. 59-71, 1998.

[20] M. J. Park and S. Nam, "Efficient calculation of the Green's function in rectangular waveguides," IEEE Int. Antennas and Propagation Symp., vol. 4, pp. 2354-2357, July 13-18, 1997.

[21] S. Singh and R. Singh, "On the use fo Shanks' transform to accelerate the summation of slowly converging series," IEEE Trans. Microwave Theory Tech., vol. 39, pp. 608-610, Mar. 1991.

[22] R. E. Collin, Field Theory of Guided Waves. New York, NY: Mc GrawHill, 1960.

[23] K. A. Michalski and J. R. Mosig, "Multilayered media Green's functions in integral equation formulations," IEEE Trans. Antennas Propagat., vol. 45, pp. 508-519, Mar. 1997.

[24] S. G. Panm and I. Wolff, "Scalarization of dyadic spectral Green's functions and network formalism for three-dimensional full-wave analysis of planar lines and antennas," IEEE Trans. Microwave Theory Tech., vol. 42, pp. 2118-2127, Nov. 1994

[25] J. R. Mosig, "Integral equation techniques," in Numerical Techniques for Microwave and Millimeter-Waves Passive Structures, T. Itoh, Ed. New York, NY: Wiley, 1989, ch. 3, pp. 133-213.

[26] J. R. Mosig and F. E. Gardiol, "Analytical and numerical techniques in the Green's function treatment of microstrip antennas and scatterers," Proc. IEE Microwave Antennas and Propagation, pt. H, vol. 130, pp. 175-182, Mar. 1983.

[27] J. R. Mosig and T. K. Sarkar, "Comparison of quasistatic and exact electromagnetic fields from a horizontal dipole above a lossy dielectric backed by an imperfect ground plane," IEEE Trans. Microwave Theory Tech., vol. MTT-34, pp. 379-387, Apr. 1986.

[28] A. Alvarez-Melcon and J. R. Mosig, "The summation by parts technique: A novel procedure for the convergence acceleration of series arising in electromagnetic problems," in Proc. XXVI General Assembly Int. Union Radio Sci., URSI'99, Toronto, ON, Canada, Aug. 1999, p. 104

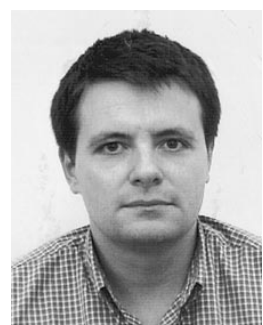

Alejandro Alvarez-Melcón was born in Madrid, Spain, in 1965. He received the Ingeniero Superior de Telecomunicaciones degree from the Polytechnic University of Madrid (UPM), Madrid, Spain, in 1991, and the Ph.D. degree in electrical engineering from the Swiss Federal Institute of Technology, Lausanne, Switzerland, in 1998.

In 1988 he joined the Signal, Systems and Radiocommunications Department, UPM, as a Research Student, where he was involved in the design, testing, and measurement of broad-band spiral antennas for electromagnetic measurements support (EMS) equipment. From 1991 to 1993, he was with the Radio Frequency Systems Division, European Space Agency (ESA/ESTEC), Noordwijk, The Netherlands, where he was involved in the development of analytical and numerical tools for the study of waveguide discontinuities, planar transmission lines, and microwave filters. From 1993 to 1995, he was with the Space Division, Industry Alcatel Espacio, Madrid, Spain, and he worked at the ESA, where he collaborated on several ESA/ESTEC contracts. From 1995 to 1999, he was with the Swiss Federal Institute of Technology, École Polytechnique Fédérale de Lausanne, Lausanne, Switzerland, where he worked in the field of microstrip antennas and printed circuits for space applications. In 2000, he joined the Polytechnic University of Carthagena, Cartagena, Spain, where he is currently developing his teaching and research activities.

Dr. Alvarez-Melcón received the JINA Best Paper Award for the best contribution to the JINA'98 International Symposium on Antennas and the COIT/AEIT Award to the best Ph.D. thesis in basic information and communication technologies.

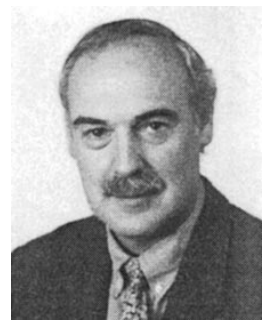

Juan R. Mosig (S'76-M'87-SM'94-F'99) was born in Cadiz, Spain. He received the electrical engineer degree in 1973 from Universidad Politecnica de Madrid, Spain. In 1976 he joined the Laboratory of Electromagnetics and Acoustics at Ecole Polytechnique Fédérale de Lausanne (EPFL), Switzerland, from which he obtained a Ph.D. degree in 1983.

Since 1991 he has been a Professor at EPFL. In 1984, he was a Visiting Research Associate at Rochester Institute of Technology, Rochester, NY. He has also held scientific appointments at universities of Rennes (France), Nice (France), Technical University of Danemark, and University of Colorado, Boulder. He is the author of four chapters in books on microstrip antennas and circuits. He is co-organizer and lecturer of yearly short intensive courses in numerical electromagnetics (Europe and USA). His research interests include electromagnetic theory, numerical methods, and microstrip antennas.

He is a member of the Swiss Federal Commission for Space Applications and responsible for several research projects for the European Space Agency. 